\title{
NOISE ANALYSIS OF A TUNNELING ACCELEROMETER BASED ON STATE SPACE STOCHASTIC THEORY
}

\author{
L.A. Oropeza-Ramos ${ }^{1}$, N. Kataria ${ }^{2}$, C.B. Burgner ${ }^{1}$, K.J. Aström ${ }^{1}$, F. Brewer ${ }^{2}$ and K.L. Turner ${ }^{1}$ \\ ${ }^{1}$ Mechanical Engineering Department ${ }^{2}$ Computer Science Department \\ University of California, Santa Barbara, CA, 93106 USA
}

\begin{abstract}
This paper presents noise analysis of a lateral tunneling accelerometer, considering Brownian motion, Nyquist-Johnson and shot noise. The tunneling accelerometer has been fabricated; a low noise differential transresistance amplifier and a digital integral controller have been designed and implemented. Noise analysis has been made based on state space models and compared with experimental results.
\end{abstract}

\section{INTRODUCTION}

The measurement of acceleration, in addition to being a central element of inertial guidance systems, has application to a wide variety of commercial problems (including automotive, medical, industrial, aeronautics and defense sectors). Different transducer mechanisms have been used in micro fabricated accelerometers [1].

Tunneling is a highly sensitive method for measuring position, which was inspired by the early work on scanning tunneling microscope. An electrostatic actuator is used, which has the advantage over capacitive, piezo-resistive and piezoelectric displacement transducers that the critical sensing area is reduced, and sensitivity and bandwidth are higher. The tunneling current is also largely independent of temperature.

\section{Motivation}

An understanding of the effects of noise is important to determine the factors that limit sensor resolution. There has been considerable work on tunneling accelerometers [2-8]. The analysis presented by Gabrielson [10] concludes that thermal noise is the main factor for most of the tunneling accelerometers. Work from JPL and Stanford reported large amounts of noise at low frequencies (below $100 \mathrm{~Hz}$ ), and their origin is still under investigation. A mathematical model to simulate the $1 / \mathrm{f}$ noise related to surface adsorption-desorption process is presented in [9]. In this paper, we use a different theoretical approach that makes it very easy to investigate the effects of different noise sources on a tunneling accelerometer. The analysis is compared the experimental data using a simple integrating controller.

\section{Principle}

In tunneling displacement transducers, the theoretical relationship between the tunneling electrode gap and the tunneling current is given by

$$
I_{t} \propto V_{b} e^{-\alpha \sqrt{\Phi} x_{o}}
$$

where $I_{t}$ is the tunneling current, $V_{b}$ is tunneling bias across the tunneling electrode gap; $\alpha$ is the tunneling constant; $\Phi$ is the effective height of the tunneling barrier or the effective work function and $x_{o}$ is the shortest tunneling gap between tunneling tip and the proof-mass electrode. Electron tunneling can only be observed when the gap between a pair of clean metal electrodes is nearly on the order of $1 \mathrm{~nm}$.

When the micro machined tunneling accelerometer is accelerated, the proof-mass experiences an inertial force that causes its motion (see Figure1). Because of the strong exponential dependence of current distance, it is necessary to use force feedback with the tip to keep a constant tunneling current. In this case, an electrostatic force is used to control the position of the proof mass. Comparing the tunneling current to a reference signal, the feedback control adjusts the electrostatic rebalance force that maintains a constant tunneling electrode separation, as is represented by the block diagram in Figure2. The feedback force is the measurement of the external acceleration.

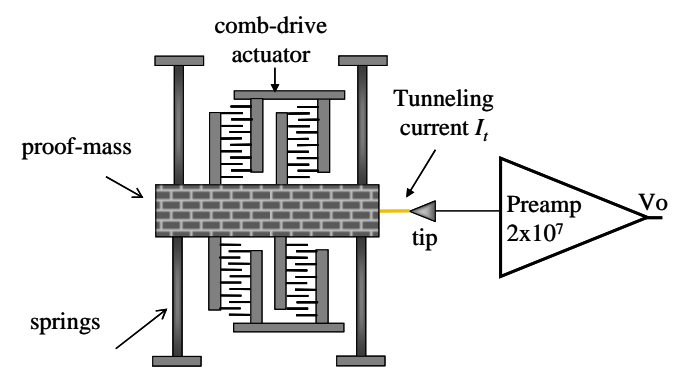

Figure 1. Schematic of a tunneling accelerometer structure.

\section{THE SYSTEM}

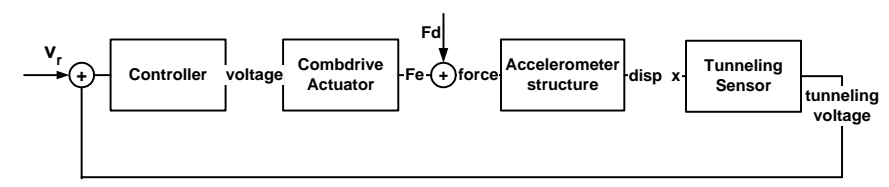

Figure 2. Schematic block diagram of the closed loop tunneling accelerometer system.

As shown in Figure1, the basic design of a lateral tunneling accelerometer consists of four elements: the proof-mass that moves relative to the substrate in response to an external stimulus, a sensor based on the tunneling tip, an electrostatic actuator with interleaved comb fingers, and folded flexural springs. The oscillatory can be simplified as a lumped model of a spring-massdamper system. The equation of motion is:

$$
m \frac{d^{2} x}{d t^{2}}+c \frac{d x}{d t}+k x=F_{e}+F_{d}
$$

where $x$ is displacement, $m$ is the mass, $k$ and $c$ are the stiffness and damping coefficients respectively, $F_{e}$ is the electrostatic force and $F_{d}$ is the external disturbance.

Our device is based on the device designed in [11]. It consists of a large proof-mass actuated laterally by an electrostatic actuation force governed by:

$$
F_{e}=N \frac{\varepsilon \cdot h \cdot}{d} V^{2}
$$

where $N$ is the number of movable fingers, $\epsilon$ is the air dielectric constant, $d$ is the space between fingers, $h$ is the depth of the fingers and $V$ is the voltage applied..

\section{NOISE ANALYSIS}

In this section the stochastic dynamical model of the closed loop system is constructed using stochastic control theory [12]. Thermo-mechanical noise from the proof-mass motion, shot noise in the tunneling junction, and Johnson-Nyquist resistor noise are considered. The analysis is based on linearized models which block diagram is shown in Figure 4. 


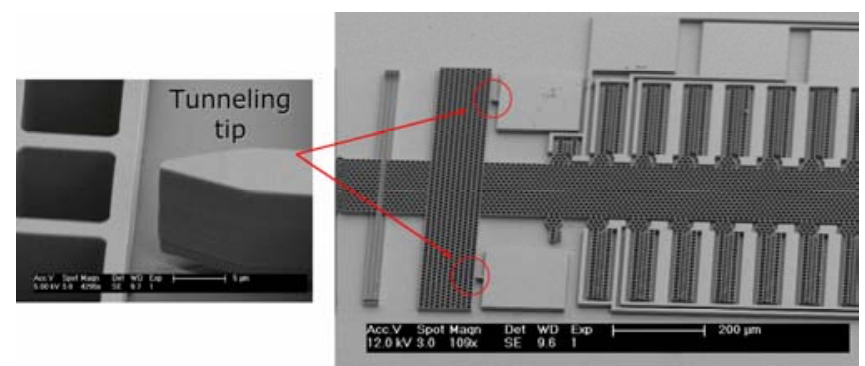

Figure 3. Electron micrograph of the large lateral tunneling accelerometer fabricated in a SOI wafer $\left(20 \mu \mathrm{m} \mathrm{Si} / 5 \mu \mathrm{m} \mathrm{SiO}{ }_{2}\right)$.

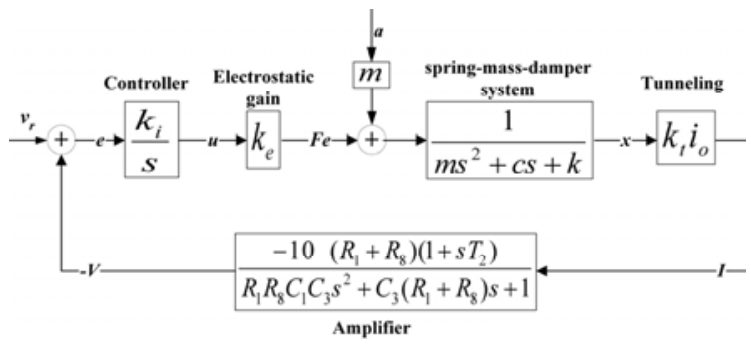

Figure 4. Block Diagram of the linearized model.

\section{Suspended Mass}

The accelerometer structure is modeled by equation (2). The force-voltage relation of the comb actuator is $F_{e}=I V^{2}$ where $\Gamma=N \epsilon h / d$. Let $V^{2}=\left(V_{o}+u\right)^{2}$, where $V_{o}$ is the bias voltage necessary to reach the tunneling operating point and $u$ is the feedback control signal. Thus $V^{2}=\left(V_{o}^{2}+2 V_{o} u+u^{2}\right)$. The middle term dominates since $V_{o} \gg u$. Introducing gain $k_{e}=2 \Gamma V_{o}$, the force $F_{e}$ in (2) is simply $F_{e}=k_{e} u$. The disturbances represented by $F_{d}$ in (2) correspond to the external acceleration and the thermal noise forces $r$ and $n_{t h}$, hence $F_{d}=r+n_{t h}$.

Introducing $a=(\mathrm{k} / \mathrm{m})^{1 / 2}$ and $c / m=2 \zeta a$ and the state variables $x_{1}=x$ and $x_{2}=\dot{x}$, the spring-mass-damper system (2) is given by:

where

$$
\dot{x}_{a}=A_{a} x_{a}+B_{a}\left(k_{e} u+r+n_{t h}\right) ; \quad y_{a}=C_{a} x_{a}
$$

$$
x_{a}=\left(\begin{array}{l}
x_{1} \\
x_{2}
\end{array}\right) ; \quad A_{a}=\left(\begin{array}{cc}
0 & 1 \\
-a^{2} & -2 \varsigma a
\end{array}\right) ; \quad B_{a}=\left(\begin{array}{c}
0 \\
\frac{1}{m}
\end{array}\right) ; \quad C_{a}=\left(\begin{array}{ll}
1 & 0
\end{array}\right)
$$

Modeling the noise as ideal white noise, the system can be described by the linear stochastic differential equation:

$$
d x_{a}=A_{a} x_{a} d t+B_{a} w_{t h}
$$

where $w_{t h}$ is a Wiener process with incremental covariance $E d w_{t h}{ }^{2}=r_{t h} d t$. The parameter $r_{t h}$ can be determined from the Boltzmann's equipartition law which gives $r_{t h}=2 c k_{B} T$, where $T$ is the absolute temperature. The corresponding spectral density is $\Phi(\omega)=c k_{B} T / \pi\left[N^{2} / \mathrm{rad} / \mathrm{s}\right]=2 c k_{B} T\left[N^{2} / \mathrm{Hz}\right]$. Notice that spectral density is proportional to the damping coefficient $c$ (dissipationfluctuation property).

\section{Tunneling Detector}

The relation between tunneling current and tip-electrode distance is given by equation (1). Linearizing it around the operating point $x_{O}=10 \AA$ gives

$$
k_{t}=\left.\frac{d I_{t}}{d x}\right|_{x=x_{o}}=\alpha \sqrt{\Phi} i_{o}
$$

$[A / m]$

The total current is then

$$
I=k_{t}\left(i_{o}\right) x+n_{t}\left(i_{o}\right)
$$

where the gain and the noise intensity depends on the nominal tunneling current $i_{o}$. The tunneling noise $n_{t}$ is attributed to the discreteness of the electrical charges, known as shot noise, which has an incremental covariance of $E\left(d w_{t}\right)^{2}=r_{t} d t$ with $r_{t}=q i_{o}\left[A^{2} / \mathrm{Hz}\right]$.

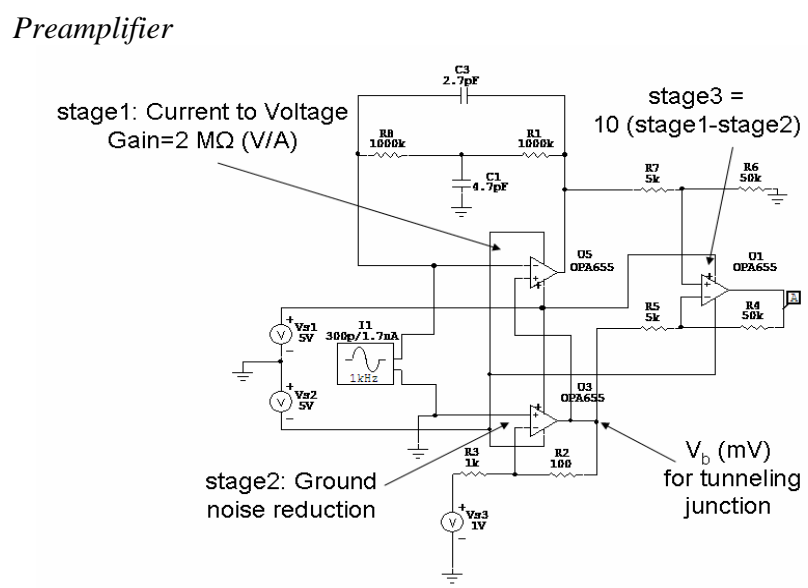

Figure 5. Circuit diagram of the differential preamplifier with a gain of $2 \times 10^{7} \mathrm{~V} / \mathrm{A}$.

A low noise transimpedance amplifier based on differential voltage sensing scheme is implemented for tunneling detection. It is designed to alleviate ground noise and ground loop problems. The circuit diagram is shown in Figure5.

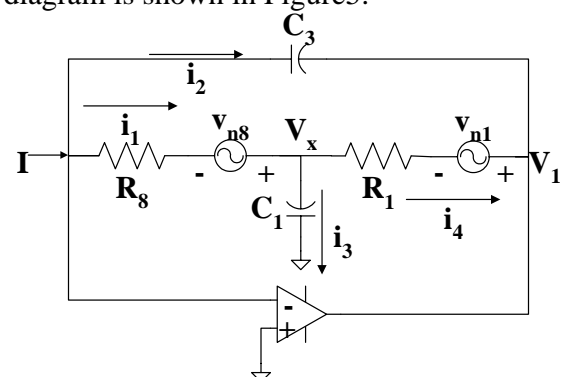

Figure 6. Equivalent non-ideaP circuit of the preamp stage1. The voltage sources represent the resistors fluctuations.

Resistors are modeled as ideal resistors with a series voltage source representing Johnson-Nyquist noise, as shown in Figure6. The noise in the first stage dominates and we will therefore only consider noise in resistors $R 1$ and $R 8$. Introduce $x_{3}=V_{x}$ and $x_{4}=10 V_{1}$ (note that the last stage of the circuit adds a gain of 10 to the output $V_{1}$ ), the state space representation of the preamplifier is given by

$$
\begin{gathered}
\dot{x}_{d}=A_{d} x_{d}+B_{d} I+B_{R} n_{R} \quad y_{d}=C_{d} x_{d} \text { where } \\
x_{d}=\left(\begin{array}{c}
x_{3} \\
x_{4}
\end{array}\right) ; A_{d}=\left(\begin{array}{cc}
-\left(\frac{1}{C 1 R 8}+\frac{1}{10 R 1 C 1}\right) & \frac{1}{R 1 C 1} \\
\frac{-10}{C 3 R 8} & 0
\end{array}\right) \quad B_{d}=\left(\begin{array}{c}
0 \\
\frac{10}{C 3}
\end{array}\right) \\
B_{R}=\left(\begin{array}{cc}
\frac{-1}{R 1 C 1} & \frac{1}{C 1 R 8} \\
0 & \frac{10}{C 3 R 8}
\end{array}\right) C_{d}=\left(\begin{array}{ll}
0 & 1
\end{array}\right)
\end{gathered}
$$

The vector $n_{R}$ represents the noise in the preamplifier which primarily is due to the resistors in the first stage. Substituting $I$ with equation (6), the tunneling detector with noise sources can be represented as a stochastic differential equation:

$$
d x_{d}=A_{d} x_{d} d t+B_{d} k_{t}\left(i_{o}\right) x_{1} d t+B_{d} d w_{t}+B_{R} d w_{R}
$$

$y_{d}=C_{d} x_{d} ; w_{t}$ and $w_{R}$ are Wiener processes with incremental covariance: $R_{d}=B_{d} r_{t} B_{d}{ }^{T}+B_{R} R_{R} B_{R}{ }^{T} ; r_{t}=q I_{t} ; \quad R_{R}=\left(\begin{array}{cc}r_{R 1} & 0 \\ 0 & r_{R 8}\end{array}\right)$ and $\quad r_{R 1}=2 k_{B} T R_{1} \quad r_{R 8}=2 k_{B} T R_{8}$. 
Controller

The integral controller can be modeled as

$$
u=k_{i} \int_{0}^{t}\left(v_{r}(\tau)-x_{4}(\tau)\right) d \tau
$$

where $k_{i}$ is determined by the design procedure in [13] and $v_{r}$ is the reference voltage corresponding to the tunneling set point. Defining a new state $x_{5}$ such that $\dot{x}_{5}=v_{r}-x_{4}$, the differential equation that describes the control action is defined as

$$
d x_{c}=-x_{4} d t+v_{r} d t \quad y_{c}=u=k_{i} x_{5}
$$

\section{The Complete System}

The realization of accelerometer, tunneling detector and controller systems, equations (5), (7) and (8), is incorporated into a state space model and its stochastic differential equation is described as:

$$
d x=A x d t+d w
$$

where $w$ is a Wiener process with incremental covariance $E d w d w^{T}=R d t$, and

$$
\begin{gathered}
R=\left(\begin{array}{ccc}
R_{a} & 0 & 0 \\
0 & R_{d} & 0 \\
0 & 0 & 0
\end{array}\right) \quad R_{a}=B_{a} r_{t h} B_{a}^{T}=\left(\begin{array}{cc}
0 & 0 \\
0 & \frac{r_{h h}}{m^{2}}
\end{array}\right) . \\
A=\left(\begin{array}{ccccc}
0 & 1 & 0 & 0 & 0 \\
-a^{2} & -2 \varsigma a & 0 & 0 & \frac{k_{e} k_{i}}{m} \\
0 & 0 & -\left(\frac{1}{C 1 R 8}+\frac{1}{C 1 R 1}\right) & \frac{1}{C 1 R 1} & 0 \\
0 & 0 & \frac{-1}{C 3 R 8} & 0 & 0 \\
0 & 0 & 0 & -1 & 0
\end{array}\right) \quad B=\left(\begin{array}{cccc}
0 & 0 & 0 & 0 \\
\frac{1}{m} & 0 & 0 & 0 \\
0 & 0 & \frac{-1}{C 1 R 1} & \frac{1}{C 1 R 8} \\
0 & \frac{-1}{C 3} & 0 & \frac{1}{C 3 R 8} \\
0 & 0 & 0 & 0
\end{array}\right)
\end{gathered}
$$

The steady state covariance of the fluctuations are given by the Lyapunov equation [12]

$$
A P+P A^{T}+R=0
$$

which can be solved numerically with a standard Matlab program.

Table 1. System parameters.

\begin{tabular}{c|c|c|c}
\hline$h=18 \mu \mathrm{m}$ & $T=293 \mathrm{~K}$ & $f_{o}=4.2 \mathrm{kHz}$ & $i_{o}=1.75 \mathrm{nA}$ \\
$m=4.917 \mu \mathrm{g}$ & $N=540$ fingers & $R 1=1 \mathrm{M} \Omega$ & $k_{t}=4.01 \mathrm{~A} / \mathrm{m}$ \\
$Q=10$ & $\epsilon_{o}=8.854 \times 10^{-12} \mathrm{~F} / \mathrm{m}$ & $R 8=1 \mathrm{M} \Omega$ & $k_{e}=9.2 \times 10^{-7} \mathrm{~N} / \mathrm{V}$ \\
$d=2.65 \mu \mathrm{m}$ & $\alpha=1.025\left[\AA^{-1} \mathrm{eV}^{0.5}\right]$ & $C_{1}=4.7 \mathrm{pF}$ & $k_{i}=45$ \\
$\Phi=0.05 \mathrm{eV}$ & $k_{B}=1.3807 \times 10^{23} \mathrm{~J} / \mathrm{K}$ & $C_{3}=10 \mathrm{pF}$ & $V_{o} \sim 14.2 \mathrm{~V}$ \\
\hline
\end{tabular}

The contributions of the different noise sources can be computed by setting corresponding parameters to zero. Thus, the standard deviation of the output of the preamplifier $\left(\sigma *=p_{44}{ }^{1 / 2}\right)$ due to each noise source is calculated and presented in Table2.

Table 2.Contribution to different noise sources (units: $\boldsymbol{m V}$ )

\begin{tabular}{c|c|c|c|c}
\hline$\sigma_{\text {th }}=3.052$ & $r_{\text {th }} \neq 0$ & $r_{t}=0$ & $r_{R 1}=0$ & $r_{R 8}=0$ \\
\hline$\sigma_{t}=0.056$ & $r_{t} \neq 0$ & $r_{t h}=0$ & $r_{R 1}=0$ & $r_{R 8}=0$ \\
\hline$\sigma_{R 1}=0.14$ & $r_{R 1} \neq 0$ & $r_{t h}=0$ & $r_{t}=0$ & $r_{R 8}=0$ \\
\hline$\sigma_{R 1}=0.17$ & $r_{R 8} \neq 0$ & $r_{t h}=0$ & $r_{t}=0$ & $r_{R 1}=0$ \\
\hline$\sigma_{\text {tot }}=3.1$ & $r_{t h} \neq 0$ & $r_{t} \neq 0$ & $r_{R 1} \neq 0$ & $r_{R 8} \neq 0$ \\
\hline
\end{tabular}

It is clear that the noise is dominated by the thermal noise. Considering contributions from all the noise sources, the fluctuations in the preamplifier output have a standard deviation of $\sigma_{\text {tot }}=3.1 \mathrm{mV}$.

To analyze the noise behavior in the frequency domain, lets consider the theorem of the spectral factorization of continuous time process [12], which establishes that a stochastic input signal with spectral density $\Phi_{\mathrm{u}}(\omega)$ propagates into a stable dynamical system $G(s)$ in such a way that the output signal has a spectral density $\Phi_{\mathrm{y}}=G(i \omega) G(-i \omega) \Phi_{u}(\omega)$. Figure7 is the plot of the spectral densities of the preamp output due to each noise source and the total sum. Notice that at low frequencies, the thermo-mechanical noise $n_{\text {th }}$ has the larger contribution but at high frequencies it is the resistor noise $n_{R 8}$ of the amplifier who dominates, as expected.

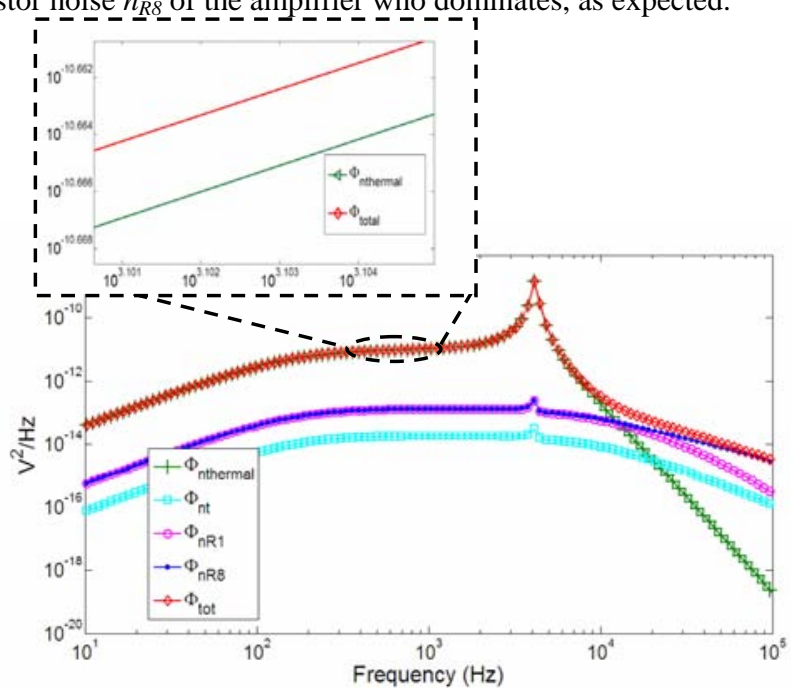

Figure 7 . Power spectral densities of the tunnneling voltage due to each noise source.

\section{EXPERIMENTAL RESULTS}

The controller is implemented in a digital platform based on Mathworks@ xPC Target prototyping.

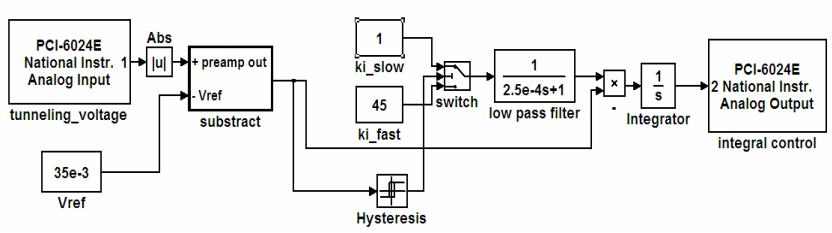

Figure 8. Simulink model of the controller implementation, including the I/O blocks that represent the analog input/output of the NI 6024E DAQ board.

Figure 8 shows the Simulink model of the system. The analog input from the DAQ board corresponds to the tunneling voltage of the preamplifier. This signal is compared to a reference of $35 \mathrm{mV}$ which corresponds to a tunneling current of $1.75 n A$. The difference between the tunneling voltage and the reference is the error signal to the controller, which is simply and integrator with gain $k_{i \_f a s t}$. This gain is used during tunneling.

To start tunneling it is necessary to move the mass gradually towards the tunneling tip. This is also accomplished using the integrating controller but with a lower gain. When the mass is far away from the tip it thus moves with constant speed given by the set point. When tunneling starts, the error signal becomes small and the gain is switched to the larger value automatically. A hysteresis has been introduced to avoid spurious switches. This simple device has proven very robust and its use is straightforward.

The smallest possible sampling time, due to hardware limitations, is $T_{s}=2 \times 10^{-4} \mathrm{sec}$. Figure 9 shows the responses of the tunneling transient and the control action $u$ during $100 \mathrm{sec}$ monitoring. There is no tunneling initially and observe that the variations in the measured signal are due to resistor noise. The control signal increases linearly and the mass approaches the tunneling tip. Tunneling begins after $50 \mathrm{sec}$ at which point the tunneling current grows exponentially towards $35 \mathrm{mV}$. The controller gain is then switched keeping the tunneling current close 
to the set point. The experiment is robust and reproducible but the noise level varies somewhat depending on the general noise level in the lab. From the curves shown in Figure9 the standard deviation of the noise is $\sigma_{\text {exp }}=0.26 \mathrm{mV}$ before tunneling starts, and $\sigma_{\text {exp }}=2.3 \mathrm{mV}$ during tunneling. Considering the gain of the preamplifier and $k_{t}$, these fluctuations correspond to variations of $0.016 \AA$ and $0.14 \AA$ before and after tunneling. The increment of the noise level is primarily due to thermal noise. The values are close to the values obtained by analysis of the model, and the difference may be due to inaccuracies of the tunneling model. It has been observed that environmental conditions change the value of the work function $\Phi$ [8], which is considered constant in the theoretical tunneling-electrode distance relation.
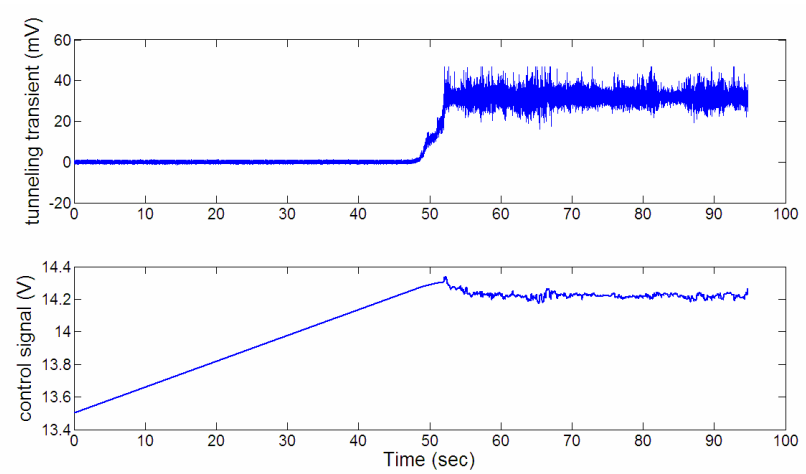

Figure 9. Exponential transient response of the tunneling signal (top) compared to the actuation voltage corresponding to the control action $u$ (bottom).

The distribution of the experimental tunneling signal is plotted in Figure10. The signal has a Gaussian distribution as is expected for a linear system with white noise excitation.

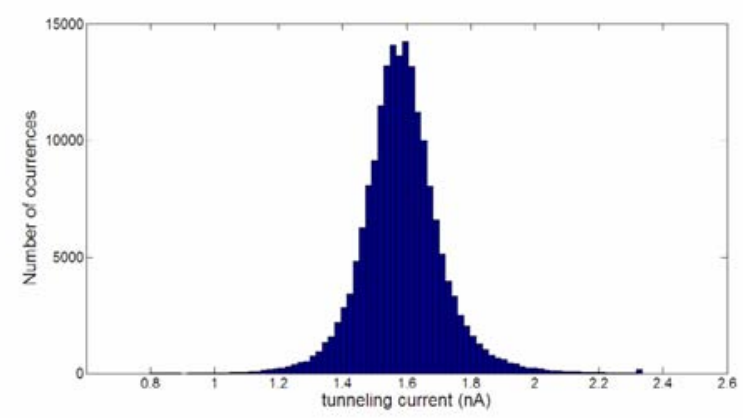

Figure 10. Histogram of the tunneling current. It is clear the signal has a Gaussian distribution as expected for white noise.

\section{Shaker Testing}

The tunneling accelerometer was placed in a Brüel\&Kjaer 4808 vibration exciter [14] to test the sensor operation. The top plot in Figure11 corresponds to the tunneling current and the bottom trace is the control signal. Notice that the integral controller is not able to overcome the complete external shaking as expected. However, this result shows the operation of our inertial sensor with a simple tracking closed loop.

\section{CONCLUSIONS}

A noise analysis of a tunneling accelerometer based on stochastic state space theory is developed. Considering Brownian, Johnson and shot noise as sources, the theoretical standard deviation of the tunneling signal is calculated to be $\sigma_{\text {tot }}=3.1 \mathrm{mV}$ and it is compared with experimental observations $\sigma_{\text {exp }}=2.3 m V$. This difference may be contributed by inaccuracies of the work function $\Phi$ in our tunneling model.

The state space analysis presented here gives a good insight of the stochastic behavior of the tunneling current and how noise propagates in the system. The controller used was a simple integrating controller which gave a fairly low bandwidth. With the current experimental set up, our results confirm Gabrielson's conclusion that thermal noise gives the major contribution. The bandwidth can be increased by using a more sophisticated controller. Such a controller will have a higher bandwidth and more resistor noise will be fed into the systems. We are currently exploring the effect of more complicated controllers that will give an instrument with higher bandwidth.

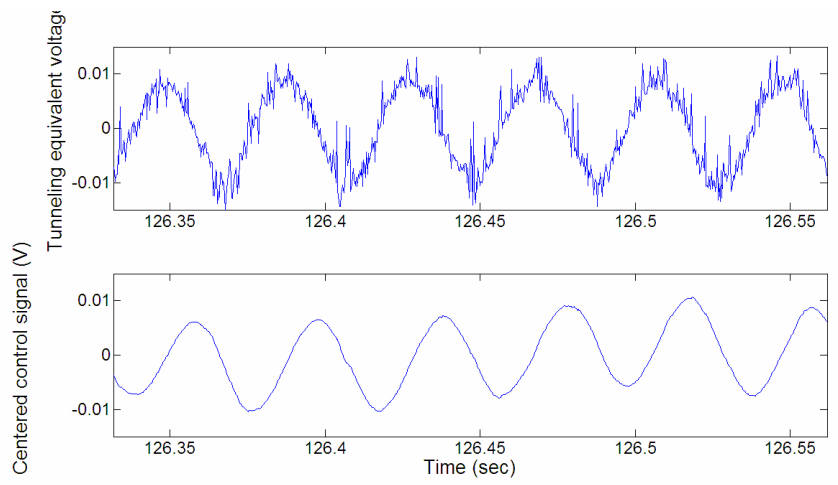

Figure 11. Shaker testing results. The input signal is a sine of 25 $\mathrm{Hz}$ frequency and $400 \mathrm{mV}$ amplitude.

\section{REFERENCES}

[1] N. Yazdi, F. Ayazi, and K. Najafi, "Micromachined inertial sensors," Proceedings of the IEEE, vol. 86, pp. 1640-1659, August 1998.

[2] T. Kenny, S. Waltman, J. Reynolds, and W. Kaiser, "A micromachined silicon electron tunneling sensor," Proceedings, An Investigation of Micro Structures, Sensors, Actuators, Machines and Robots, pp. 192196, February 1990.

[3] — , "Micromachined silicon tunnel sensor for motion detection," Appl. Phys. Lett, vol. 58, pp. 100-102, January 1991.

[4] H. Rockstad, T. Kenny, J. Reynolds, W. Kaiser, and T. Gabrielson, “A miniature high-sensitivity broad-band accelerometer based on electron tunneling transducer," Sensors and Actuators A, vol. 43, January 1994.

[5] H. Rockstad, J. Reynolds, T. Tang, T. Kenny, W. Kaiser, and T. Gabrielson, "A miniature, high-sensitivity, electron tunneling accelerometer,” Transducers'95, vol. 2, June 1995.

[6] P. Scheeper, J. Reynolds, and T. Kenny, "Development of a modal analysis accelerometer based on a tunneling displacement transducer,” Transducers'97, vol. 2, pp. 867-870, June 1997.

[7] C. Yeh and K. Najafi, "A low-voltage tunneling-based silicon microaccelerometerl," Transactions on Electronic Devices, IEEE, vol. 44, pp. 1875-1882, Nov 1997.

[8] P. Hartwell, F. Bertsch, S. Miller, K. Turner, and N. MacDonald, "Single mask lateral tunneling accelerometer," Proceedings of MEMS'98, pp. 340-344, Jan 1998.

[9] J. Wang, P. Zavracky, N. McGruer, and R. Morrison, "Study of tunneling noise using surface micromachined tunneling tipdevices," Transducers'97, vol. 1, pp. 467-470, June 1997.

[10] T. Gabrielson, "Mechanical-thermal noise in micromachined acoustic and vibration sensors," IEEE transactions on Electron Devices, vol. 40, pp. 903-909, May 1993.

[11] P. Hartwell, "Velocity and displacement measurements of microelectromechanical systems using laser vibrometry,” $\mathrm{PhD}$ Thesis, Cornell University, August 1999.

[12] K. J. Åström, Introduction to Stochastic Control Theory. Dover, 1970.

[13] K. J. Åström and R. M. Murray, Feedback Systems. An Introduction for Scientists and Engineers. Princeton University Press, 2008.

[14 ] www.bksv.com 